\title{
LATTICES OF NORMALLY INDECOMPOSABLE MODULES
}

\author{
JULIUSZ BRZEZINSKI
}

\begin{abstract}
If $M, N$ are finitely generated left $R$-modules, then $M$ divides $N^{1}$ if there is an epimorphism $M^{(r)} \rightarrow N$. M is normally indecomposable if $M \simeq M_{1} \oplus M_{2}$ and $M_{1}$ divides $M_{2}$ imply $M_{2}=0$. If $R$ is an Artin algebra or an order over a complete discrete valuation ring in a semisimple algebra, the set of isomorphism classes of normally indecomposable $R$-modules (respectively $R$-lattices) is partially ordered by the divisibility relation. We show that for $R$ of finite representation type this partially ordered set is a lattice satisfying the Jordan-Dedekind chain condition and the length of maximal chains is equal to the number of isomorphism classes of indecomposable $R$-modules (respectively $R$-lattices).
\end{abstract}

1. Introduction. Let $R$ be an associative ring and $M, N$ two finitely generated left $R$-modules. We say that $M$ divides $N$ (notation: $M>N$ ) if there is an epimorphism $M^{(r)} \rightarrow N$, where $M^{(r)}$ denotes the direct sum of $r$ copies of $M$ (see Roiter [5]). An $R$-module $M$ is indecomposable if $M \cong M_{1}$ $\oplus M_{2}, M \neq 0$ imply $M_{1}=0$ or $M_{2}=0$ and normally indecomposable (see [5]) if $M \cong M_{1} \oplus M_{2}, M_{1} \geqslant M_{2}$ imply $M_{2}=0$. Let $(5)$ be an additive full subcategory of the category of finitely generated left $R$-modules such that $R$ (as a left $R$-module) is in $\mathbb{E}$ and every direct summand of a module in $\mathbb{E}$ is in $\Subset$. We say that $\mathbb{E}$ is a Krull-Schmidt category (a $K S$-category) if the KrullSchmidt theorem holds in $\mathbb{E}$, that is, every nonzero module in $\mathbb{E}$ is a direct sum of indecomposable $R$-modules and the summands are uniquely determined up to order and $R$-isomorphism. If there is only a finite number of isomorphism classes of indecomposable modules in $\mathbb{E}$, we say $\mathbb{E}$ has finite type. The relation " $\geqslant$ " defines a quasi-ordering of the set of isomorphism classes of $R$-modules. We say that $\mathbb{E}$ is normal if this relation is a partial ordering of the set of isomorphism classes of normally indecomposable $R$-modules in $\mathbb{E}$, that is, $M \geqslant N, N \geqslant M, M, N$ in $\sqrt{ }$ and normally indecomposable imply $M \cong N$. Our aim is to prove that if $\mathbb{E}$ is a normal $K S$-category of finite type, then the isomorphism classes of normally indecomposable objects in $\mathbb{E}$ form a lattice satisfying the Jordan-Dedekind chain condition, that is, all maximal chains between fixed end points have the same length (see [1, p. 11]). The length of such a chain between the least element

Received by the editors March 29, 1977.

AMS (MOS) subject classifications (1970). Primary 16A64, 06A20; Secondary 16A46, 18B99.

Key words and phrases. Krull-Schmidt theorem, normal decomposition of modules, JordanDedekind chain condition, finite representation type.

'We use the term "divide" (introduced by Roiter), since the now accepted term "cover" (introduced later by Reiner) is frequently used in the paper in the sense of lattice theorv. 
(the class of $M=0$ ) and the greatest element (the class of a normally indecomposable $R$-progenerator) is equal to the number of isomorphism classes of indecomposable $R$-modules in $\mathbb{E}$ (Theorem 1). Two important examples of normal $K S$-categories of finite type are the category of all finitely generated modules over an Artin algebra $R$ of finite representation type and the category of $R$-lattices, where $R$ is an $A$-order of finite representation type in a semisimple algebra over the field of fractions of a complete discrete valuation ring $A$.

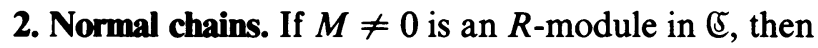

$$
M \cong M_{1} \oplus M_{2} \oplus \cdots \oplus M_{r}
$$

where $M_{1} \geqslant M_{2} \geqslant \cdots \geqslant M_{r}, M_{r} \neq 0$ and $M_{i}$ are normally indecomposable. In fact, if $M$ is normally decomposable, then $M \cong M_{1} \oplus M_{1}^{\prime}$, where $M_{1}^{\prime} \neq 0, M_{1}$ is normally indecomposable, and the minimal number of generators of $M_{1}^{\prime}$ over $R$ is less than this number for $M$, so our claim follows by induction. If $\mathbb{E}$ is a normal $K S$-category, then the decomposition (1) (in the sequel called normal) is unique up to isomorphism, since $M \cong M_{1}^{\prime} \oplus M_{2}^{\prime}$ $\oplus \cdots \oplus M_{s}^{\prime}$, where $M_{1}^{\prime} \geqslant M_{2}^{\prime} \geqslant \cdots \geqslant M_{s}^{\prime}, M_{s}^{\prime} \neq 0, M_{i}^{\prime}$ normally indecomposable give $M_{1} \geqslant M \geqslant M_{1}^{\prime}$ and $M_{1}^{\prime} \geqslant M \geqslant M_{1}$. Hence $M_{1} \cong M_{1}^{\prime}$ and by the $K S$-property we can cancel $M_{1}, M_{1}^{\prime}$ and then proceed by induction.

If $\mathfrak{E}$ is normal, then for every pair of $R$-modules $M, N$ there is a uniquely defined up to isomorphism normally indecomposable module $M \cup N$ which is "a least upper bound" of $M$ and $N$ with respect to the relation " $\geqslant$ "-if we take $M \cup N$ equal to the first direct summand in a normal decomposition of $M \oplus N$, then $M \cup N \geqslant M, M \cup N \geqslant N$ and $X \geqslant M \cup N$ if $X \geqslant M$, $X \geqslant N$.

We write $M>N$ to denote $M \geqslant N$ and $M \npreceq N$. By a normal chain we shall mean a chain of normally indecomposable modules. A finite normal chain of length $n, M_{0}>M_{1}>\cdots>M_{n}$ is called maximal if there is not a longer normal chain in $\sqrt{5}$ containing it as a subchain. We write $N \mid M$ if $M \cong N \oplus N^{\prime}$.

Proposition 1. Let $\mathbb{1}$ be a normal KS-category. The following conditions are equivalent:

(i) $\mathbb{6}$ is a category of finite type,

(ii) ascending and descending normal chains in 15 are finite,

(iii) every finite normal chain can be embedded in a maximal one,

(iv) there is at least one maximal normal chain.

Proof. (i) $\Rightarrow$ (ii) follows from the fact that a normally indecomposable module is a direct sum of nonisomorphic indecomposable modules (but, of course, such a direct sum need not be normally indecomposable). (ii) $\Rightarrow$ (iii) is true for arbitrary partially ordered sets and (iii) $\Rightarrow$ (iv) is evident. To prove (iv) $\Rightarrow$ (i), let $M_{1}>M_{2}>\cdots>M_{r}$ be a maximal normal chain and $N$ an indecomposable module. We shall prove that there is a module $M_{i}$ such that $N \mid M_{i}$. Let $N \nmid M_{i}$ for all $i$. Since $M_{1} \cup N \geqslant M_{1}$ and the chain is maximal, we 
get $M_{1} \cup N \cong M_{1}$. Hence $M_{1} \geqslant N$. If $M_{i} \geqslant N$ for $1<i<r$, then $M_{i} \geqslant$ $M_{i+1} \cup N \geqslant M_{i+1}$ and we get $M_{i+1} \cup N \cong M_{i+1}$, since $M_{i+1} \cup N \cong M_{i}$ is impossible by the assumption $N \nmid M_{i}$. Therefore $M_{i+1} \geqslant N$. But this implies $M_{r} \geqslant N$-a contradiction. Now by the $K S$-property $\&$ is of finite type.

COROLlary. If $M_{1}>M_{2}>\cdots>M_{r}$ is a maximal normal chain in a normal $K S$-category $\mathbb{E}$ and $N$ is an indecomposable module in $\sqrt{ }$, then there is $i$ such that $N \mid M_{i}$.

Let $\mathfrak{R}(\mathbb{E})$ be the set of isomorphism classes $[M]$ of normally indecomposable modules $M$ in $\mathfrak{E}$. If $\mathbb{E}$ is a normal category, then $\mathfrak{N}(\mathbb{E})$ is partially ordered by the relation " $\geqslant "$. It is easy to see that the class $[M \cup N]$ is a least upper bound $[M] \cup[N]$ of $[M]$ and $[N]$. If, moreover, every ascending normal chain in $\mathbb{E}$ is finite, there also exists a greatest lower bound $[M] \cap$ $[N]$ : the set of $[X]$ such that $[M] \geqslant[X],[N] \geqslant[X]$ is nonempty ([0] belongs to it), and if $\left[X_{1}\right],\left[X_{2}\right]$ are two maximal elements of this set, then $\left[X_{1} \cup X_{2}\right]=$ $\left[X_{1}\right]=\left[X_{2}\right]$, since $M \geqslant X_{1} \cup X_{2} \geqslant X_{i}$ and $N \geqslant X_{1} \cup X_{2} \geqslant X_{i}$. Hence $\mathfrak{\Re ( ङ )}$ is a lattice.

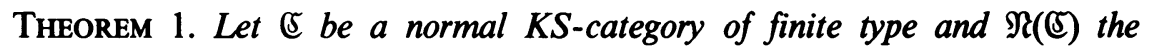
lattice of isomorphism classes of normally indecomposable modules in $\mathbb{}$. Then $\mathfrak{R}(\mathbb{E})$ satisfies the Jordan-Dedekind chain condition and the length of every maximal chain in $\mathfrak{R}(\mathfrak{E})$ is equal to the number of isomorphism classes of indecomposable modules in $(5$.

Proof. Let $d([M])=$ the number of isomorphism classes of indecomposable modules $X$ in $\mathbb{5}$ such that $M \geqslant X$. We want to prove that if $[M]>[X]$ $\geqslant[N],[M] \neq[N]$ imply $[X]=[M]$ or $[X]=[N]$ (that is, $[M]$ covers $[N]$ in the sense of partially ordered sets), then

$$
d([M])=d([N])+1 \text {. }
$$

This equality gives the first part of Theorem 1 (see [1, p. 11]). The second part follows from the fact that in every maximal normal chain the first term is an $R$-progenerator $P$ and $d([P])=$ the number of isomorphism classes of indecomposable $R$-modules in $\mathfrak{E}$, while the last term is always the zero module for which $d([0])=0$. To prove the equality (2), we need the following result:

LEMMA 1. If $M$ and $N$ are normally indecomposable in a normal KS-category (5) and $[M]$ covers $[N]$ in $\mathfrak{R}(\mathfrak{E})$, then there is precisely one indecomposable direct summand of $M$ which is not a direct summand of $N$.

Proof of Lemma 1. Let $M \cong M_{1} \oplus \cdots \oplus M_{r}, M_{i}$ indecomposable. There is at least one $j$ such that $N \ngtr M_{j}$. Hence $N \cup M_{j} \cong M$, so that $M \mid N \oplus M_{j}$ and Lemma 1 is proved.

Now, if $M \geqslant X, X$ indecomposable, and $N \geqslant X$, then $M>N \cup X>N$ gives $N \cup X \cong M$. Hence $X$ is the direct summand of $M$ that is not a direct summand of $N$. Since, of course, $N \geqslant X$ implies $M \geqslant X$, we get the required 
equality (2) and we complete the proof of Theorem 1.

3. Examples. Let $\mathbb{C}$ be an additive category such that, besides all the assumptions formulated in the Introduction, for every module $M$ in $\mathbb{E}$ the ring $\operatorname{End}_{R}(M)$ is semiperfect (a ring $A$ is semiperfect if $A / J(A), J(A)$ the Jacobson radical of $A$, is semisimple in the classical sense and every idempotent of $A / J(A)$ can be lifted to an idempotent of $A)$. $(5$ is a normal $K S$-category. The $K S$-property is equivalent to the Krull-Schmidt theorem which holds in this case, while $\mathbb{E}$ is normal by the following result:

LeMma 2 (RoITER [5, TheOREM 1]). Let $R$ be an arbitrary ring, $X \rightarrow Y \rightarrow 0$ an epimorphism of finitely generated left $R$-modules such that $Y \geqslant X$ and $Y$ is normally, indecomposable. If $\operatorname{End}_{R}(Y)$ is semiperfect, then the sequence $X \rightarrow Y$ $\rightarrow 0$ splits.

Now, if $M \geqslant N$ and $N \geqslant M$, where $M, N$ are normally indecomposable, then the sequences $M^{(r)} \rightarrow N \rightarrow 0$ and $N^{(s)} \rightarrow M \rightarrow 0$ split. Since $M, N$ are direct sums of nonisomorphic indecomposable $R$-modules, we get $M \cong N$ by the Krull-Schmidt theorem.

There are two important special cases:

(a) $R$ an Artin $A$-algebra of finite representation type, where $A$ is a commutative Artin ring,

(b) $R$ an $A$-order of finite representation type in a semisimple algebra over the field of fractions of a complete discrete valuation ring $A$.

In the first case the category of all finitely generated $R$-modules, and in the second, the category of $R$-lattices (i.e. finitely generated $A$-free $R$-modules) are normal $K S$-categories of finite type.

To describe $\mathfrak{N}(\mathbb{E})=\mathfrak{N}$ call this lattice decomposable if $\mathfrak{N}=\mathfrak{R}_{1} \cup \mathfrak{R}_{2}$, where $\mathfrak{N}_{1} \cap \mathfrak{N}_{2}=\{[0]\}$ and $M_{1} \oplus M_{2}$ is normally indecomposable for every $\left[M_{1}\right] \in \mathfrak{N}_{1}$ and $\left[M_{2}\right] \in \mathfrak{N}_{2}$. It is easy to see that $\mathfrak{N}_{1}, \mathfrak{N}_{2}$ are lattices which satisfy the Jordan-Dedekind chain condition if $\mathfrak{N}$ does. Denoting by $d(\mathfrak{N})$, $d\left(\mathfrak{N}_{i}\right)$ the length of maximal chains in $\mathfrak{N}, \mathfrak{N}_{i}$ respectively, we have

$$
d(\mathfrak{N})=d\left(\mathfrak{N}_{1}\right)+d\left(\Re_{2}\right)
$$

If $\mathfrak{N}_{i}$ has $n_{i}$ elements, then $\mathfrak{R}$ has $n_{1} n_{2}$ elements and

$$
M_{1} \oplus M_{2} \geqslant M_{1}^{\prime} \oplus M_{2}^{\prime}
$$

where $\left[M_{i}\right],\left[M_{i}^{\prime}\right] \in \mathfrak{N}_{i}$ if and only if $M_{i} \geqslant M_{i}^{\prime}$. To prove the last statement, let us note that $\left[M_{1} \cup M_{1}^{\prime}\right] \in \Re_{1}$ and $\left(M_{1} \cup M_{1}^{\prime}\right) \oplus M_{2} \geqslant M_{1} \oplus M_{2}$. Hence $\left(M_{1} \cup M_{1}^{\prime}\right) \oplus M_{2} \cong M_{1} \oplus M_{2}$ and by the $K S$-property $M_{1} \cup M_{1}^{\prime} \cong M_{1}$. Therefore $M_{1} \geqslant M_{1}^{\prime}$. In the same way $M_{2} \geqslant M_{2}^{\prime}$. Let us note that every maximal chain in $\mathfrak{R}(\mathfrak{E})$ is a kind of composition series of the top element and every step down corresponds to an indecomposable module (Lemma 1). It is easy to prove that indecomposable modules correspond to the points covering (in the sense of lattice theory) only one element of the lattice.

Let us look at some examples. If $R$ is semisimple and $\mathbb{E}$ is the category of all finitely generated left $R$-modules, then $\mathfrak{R}(\mathbb{E})$ has $2^{r}$ elements, where $r$ is the 
number of nonisomorphic indecomposable (= simple) modules. In this case $\mathfrak{R}(\mathbb{E})=\mathfrak{N}_{1} \cup \cdots \cup \mathfrak{R}_{r}$, where every $\mathfrak{N}_{i}$ has two elements- $[0]$ and $\left[S_{i}\right], S_{i}$ a simple module. $\mathfrak{R}(\mathfrak{E})$ is isomorphic with the lattice of subsets of a set with $r$ elements. More generally, if $\mathbb{E}$ is a $K S$-category of finite type, then $\mathfrak{R}(\mathbb{E})$ is a Boolean algebra if and only if all the modules in $\mathbb{E}$ are projective.

Let $R$ be the algebra of matrices

$$
\left[\begin{array}{lll}
k & k & 0 \\
0 & k & 0 \\
0 & k & k
\end{array}\right]
$$

over a field $k$ (the algebra corresponding to the diagram $0 \rightarrow 0 \leftarrow 0$ in the sense of $[3$, p. 96]). If $\mathbb{C}$ is the category of all finitely generated $R$-modules, then $\mathfrak{R}(\mathfrak{E})=\mathfrak{N}_{1} \cup \mathfrak{N}_{2}$, where $d\left(\mathfrak{N}_{1}\right)=1, d\left(\mathfrak{R}_{2}\right)=5, \mathfrak{R}_{1}$ has $2, \mathfrak{N}_{2} 12$ elements, and the diagram of the lattice $\Re_{2}$ is (we use the notation of $[2$, p. 78] to denote the indecomposable $R$-modules):

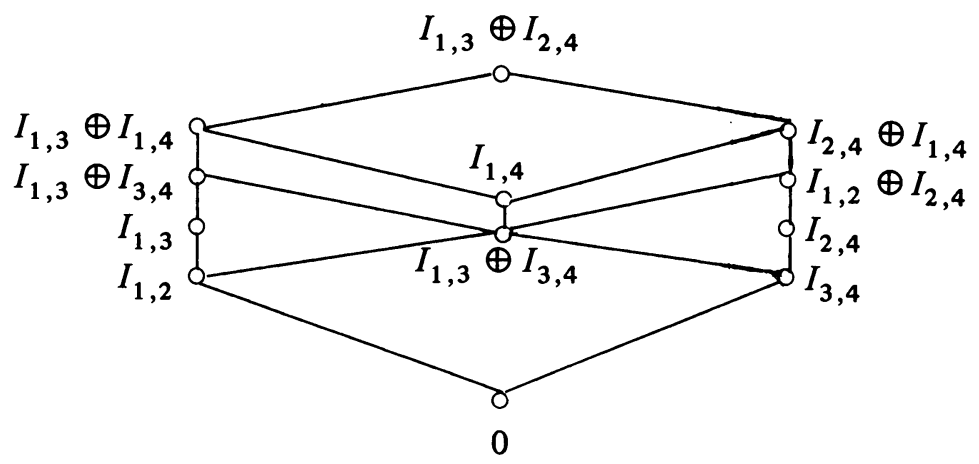

Some natural questions arise. If $R=k\{t\}$, the ring of formal power series, there is an infinite ascending normal chain $M_{1}<M_{2}<\cdots<M_{r}<\ldots$, where $M_{r}=k\{t\} /\left(t^{r}\right)$, but every descending normal chain is finite. If $R=k$ $\bigoplus k x \oplus k y$, where $x^{2}=y^{2}=x y=y x=0$, there are infinite ascending and descending normal chains. We can take (see [4, Proposition 5]) $M_{r}=V \oplus W$, where

$$
V=k a_{1} \oplus \cdots \oplus k a_{r}, \quad W=k b_{0} \oplus \cdots \oplus k b_{r}
$$

and $x a_{i}=b_{i}, y a_{i}=b_{i-1}$ for $1 \leqslant i \leqslant r, x b_{i}=y b_{i}=0$ for $0 \leqslant i \leqslant r$. The modules $M_{r}$ are indecomposable. It is easy to see that there is an epimorphism $M_{r}^{(2)} \rightarrow M_{r+1}$, so $M_{r}>M_{r+1}$ for $r=1,2, \ldots$ On the other hand, $M_{r} \subset M_{r+1}$ and we get an ascending normal chain, since $M_{r+1}^{*}>M_{r}^{*}$ for $r=1,2, \ldots$, where $M_{r}^{*}=\operatorname{Hom}_{k}\left(M_{r}, k\right)$.

A question is if one of the chain conditions over an Artin algebra implies the other (and the finite representation type as a consequence). Let us note that $\mathfrak{R}(\mathbb{E})$ is a lattice if the ascending normal chains in $\mathbb{E}$ are finite. Is $\mathfrak{R}(\mathbb{E})$ finite in this case?

ADDED IN PROOF. Over an Artin algebra finiteness of ascending normal chains implies finiteness of descending normal chains. This is an easy 
consequence of the main theorem of M. Auslander's paper Large Modules over Artin Algebras in A Collection of Papers in Honour of Samuel Eilenberg, Academic Press, New York, 1976. The same fact in the case of orders over complete valuation rings follows from a theorem of C. M. Ringel and K. W. Roggenkamp in Indecomposable Representations of Orders and Dynkin Diagrams (to appear).

\section{REFERENCES}

1. G. Birkhoff, Lattice theory, 3rd ed., Amer. Math. Soc. Colloq. Publ., vol. 25, Amer. Math. Soc., Providence, R.I., 1967. MR 37 \#2638.

2. P. Gabriel, Unzerlegbare Darstellungen. I, Manuscripta Math. 6 (1972), 71-103. MR 48 \#11212.

3.

Indecomposable representations. II, Symposia Mathematica, Vol. 11 (INDAM, Roma, 1971), Academic Press, London, 1973, pp. 81-104. MR 49 \#51 32

4. A. Heller and I. Reiner, Indecomposable representations, Illinois J. Math. 5 (1961), 314-323. MR 23 \#A222.

5. A. V. Roiter, Divisibility in categories of representations over a complete Dedekind domain, Ukrain. Mat. Z. 17 (1965), 124-129. MR 33 \#5699.

Department of Mathematics, Chalmers University of Technology, 40220 Gothenburg, SWEDEN 